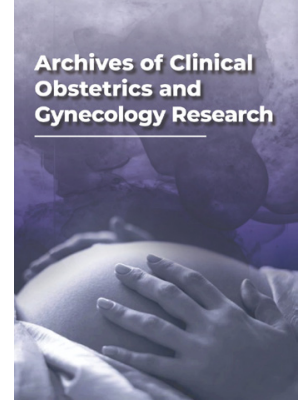

Correspondence

Xu Yefen

Tibet Agricultural and Animal Husbandry College, 100 Yucai Road, Bayi District, Nyingzhi City, Tibet, P. R. China,

Tel.: +86-0894-5822924

Fax: +86-0894-5822924

E-mail:xzlzxyf@163.com

\footnotetext{
- Received Date: 13 Nov 2020

- Accepted Date: 23 Nov 2020
}

- Publication Date: 12 Dec 2020

Keywords

Yak; GCs; BMP15; FSHR; miR-31; P4; proliferation

Copyright

(c) 2020 Science Excel. This is an openaccess article distributed under the terms of the Creative Commons Attribution 4.0 International license.

\title{
BMP15/miR-31/FSHR axis regulates yak ovarian granulosa cell proliferation and P4 secretion
}

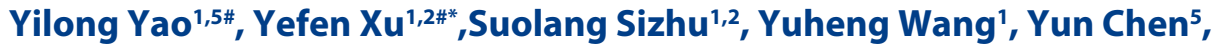 \\ Qiangba Yangzong ${ }^{1}$, Jiaqiang Niu' ${ }^{1,2}$, Honghui Wang ${ }^{1}$, Qifa $\mathrm{Li}^{5}$, Linghua Cheng ${ }^{4}$, \\ Min Guo ${ }^{4}$ \\ 'Department of Animal Science, Tibet Agricultural and Animal Husbandry College, Nyingzhi, Tibet 860000, China \\ ${ }^{2}$ Provincial Key Laboratory of Tibet Plateau Animal Epidemic Disease Research,Tibet Agricultural and Animal Husbandry College, \\ Nyingzhi, Tibet 860000 , China
}

${ }^{3}$ Ministry of Agriculture Key Laboratory of Animal Genetics, Breeding and Reproduction, National Engineering Laboratory for Animal Breeding, College of Animal Sciences and Technology, China Agricultural University, Haidian, Beijing 100193, China ${ }^{4}$ College of Animal Science and Technology, Nanjing Agricultural University, Nanjing, Jiangsu 210095, China

${ }^{5}$ Agricultural Genomics Institute at Shenzhen, Chinese Academy of Agricultural Sciences, Shenzhen, Guangzhou 518000, China "The first two authors equally contributed in the study.

\begin{abstract}
Background: Bone morphogenetic protein 15 (BMP15) is an oocyte secretory factor expressed mainly in mammalian oocytes and exerts biological functions through binding to specific receptors on the granulosa/sheath cell membrane. BMP15 regulates the development and proliferation of granulosa cells (GCs) and inhibits follicle stimulating hormone (FSH)-dependent cell differentiation and proliferation. In this report, the regulatory mechanism of BMP15 on yak ovary granulosa cell proliferation and hormone secretion was examined. Methods: An iodine progesterone radio-immunoassay was used to detect the secretion of progesterone (P4) in yak granulosa cells. FSH receptor (FSHR) binding miRNAs were predicted by bioinformatics analysis. The expression of FSHR mRNA and microRNAs was measured by qPCR, and the expression of FSHR was detected by western blotting. The proliferation of granulosa cells was measured using the CCK-8 kit. Results: We demonstrate for the first time that BMP15 downregulates the expression of FSHR and upregulates the expression of microRNA-31 (miR31) in yak GCs. Dual luciferase activity results indicate that miR-31 binds directly to the FSHR 3' UTR. miR-31 was found to promote the proliferation of yak GCs and inhibit the secretion of P4 by inhibiting the expression of the FSHR in yak GCs. Conclusions: The BMP15/miR-31/FSHR axis identified in this study is a new mechanism for regulating yak GC function.
\end{abstract}

\section{Introduction}

Yaks live mainly in cool climates above 3,000 meters sea level in the Qinghai-Tibet Plateau of China and have adapted to both cold temperatures and high altitudes. Yaks are labor-intensive animals, and are particularly important for the economic, cultural and social development of Tibet. Yaks have low fertility and thus it is very important to explore the mechanism of follicular development. The follicle stimulating hormone (FSH) is a glycoprotein gonadotropin synthesized and secreted by basophilic cells of the anterior pituitary of animals[1,2]. FSH is a heteromer composed of two non-covalently linked subunits, the alpha and beta subunits, and plays an important role in the hypothalamic pituitary gonadal axis[3,4]. In female animals, FSH promotes the differentiation of follicle intima cells, proliferation of granulosa cells (GCs) and secretion of follicular fluid $[5,6]$.

The FSH receptor (FSHR) is located on the surface of GCs and Sertoli cells $[7,8]$. In ovaries, FSHR plays key roles in regulating proliferation, differentiation, apoptosis and hormone synthesis of GCs [9-11]. Furthermore, FSH combines with
FSHR to regulate ovarian follicle growth, GC proliferation, estrogen and progesterone synthesis and secretion [12]. In addition, there is a large body of evidence showing that oocytes secrete factors that modulate FSH activity $[13,14]$. Oocytes secrete factors that inhibit FSH-induced expression of progesterone (P4) [15] and estradiol (E2) production [16]. Growth and differentiation factor-9 (GDF-9) is an oocyte factor involved in regulating these cellular proliferative and differentiation responses [17]. There is compelling evidence that bone morphogenetic protein 15 (BMP15) is closely related to GDF9 and shares several expression control mechanisms [18], such as promoting the proliferation of GCs in rats, downregulating FSH production and inhibiting the secretion of P4 [19]. In addition, Otsuka et al. showed that BMP15 downregulates FSH activity by inhibiting the expression of FSHR, regulating rat GCs development and P4 secretion [20]. In combination, these observations suggest that BMP15 activity is closely linked to FSH and FSHR expression in ovarian follicle development in mammals. However, the detailed mechanism of how BMP15 regulates the expression of FSHR in GCs of yaks remains unclear. 
MicroRNAs (miRNAs) are small non-coding RNAs of 20-22 nucleotides in length that play key roles in the ovary and regulation of GC apoptosis $[21,22]$. Recent studies showed that the androgen receptor and miR-126* target the FSHR by inhibiting FSHR expression and promoting GC apoptosis [23]. Other studies also showed that bone morphogenetic protein receptor type II, as a target of miR-375, could regulate proliferation and apoptosis of GCs by affecting BMP15 expression [24]. However, the relationship between BMP15, miRNAs and FSHR in GCs has not been fully elucidated. In this study, we identified miR-31 as a miRNA upregulated by BMP15 in yak GCs and the candidate regulatory miRNA of FSHR. The aim of this study was to explore the molecular mechanism of how BMP15 regulates FSHR and $\mathrm{P} 4$ secretion through miRNA in yak ovary GCs.

\section{Materials and Methods}

\section{Animals}

A total of twenty-five female yaks weighing $250-300 \mathrm{~kg}$ and 6-7 years of age were selected from the Livestock farms in Linzhi, situated at $2750 \mathrm{~m}$ above sea level in Linzhi, Tibet. The animals were slaughtered and healthy yak ovaries were obtained. The animals were healthy and free from any anatomical reproductive disorders and had completed a two-year postpartum period. The yak ovaries were harvested and stored in physiological saline at $38^{\circ} \mathrm{C}$ before experimental analysis.

\section{Cell culturing}

Yak GCs were isolated from 3-5 mm follicles of fresh yak ovaries. GCs and HEK293 cells were cultured in Dulbecco's Modified Eagle's medium (DMEM, Sigma-Aldrich, St Louis, MO, USA) that contained $10 \%$ fetal bovine serum (FBS, Atlanta Biologicals, Lawrenceville, GA, USA), $1 \%$ penicillin (Atlanta Biologicals), $100 \mathrm{mg} / \mathrm{mL}$ streptomycin and $1 \%(w / v)$ glutamine (Atlanta Biologicals). GCs and HEK293 cells were incubated in $5 \% \mathrm{CO}_{2}$ at $37^{\circ} \mathrm{C}$. All animal experiments were approved by the Animal Ethics Committee at Xizang Agricultural and Animal Husbandry College, China.

\section{Plasmids}

The coding sequence region of the yak FSHR gene was amplified by PCR using forward and reverse primers containing BamH I and Xho I sites, respectively. The PCR product was inserted into the pcDNA3.1 vector (Invitrogen, Nanjing, China) to create the protein overexpression plasmid. To verify whether BMP15 is a target of miR31, the FSHR 3' UTR was amplified and inserted into the pmirGLO vector (Promega, Shanghai, China) between the Sac I and Xho I sites. The vector was then identified by sequencing and the Xho I/ Sac I double restriction enzyme method. Mutant type plasmids were constructed using a point mutagenesis kit (TaKaRa, Dalian, China). Primer sequences are listed in Table 1.

\section{Cell transfection}

Cells were grown to $75-80 \%$ confluence in 12 -well or 6-well plates and then transfected with plasmids, miRNA mimic, mimic inhibitor, siRNAs or controls by using the Lipofectamine 2000 reagent (Invitrogen, Carlsbad, CA, USA), according to the manufacturer's instructions. The synthetic miR-31 mimic, negative control, FSHRspecific siRNAs (F-siRNA-1 and F-siRNA-2) and siRNA-control were obtained from Genepharma (Jima, Shanghai, China) (Table 2).

\section{Western blotting}

Western blotting was performed as described previously [25]. Antibodies against FSHR (ab75200) and GAPDH (ab8245) were obtained from Abcam (Cambridge Science Park, UK).

\section{CCK-8 analysis of yak GC proliferation}

Cells were seeded at $1.0 \times 104$ cells/well in a 96-well plate. Each well had $100 \mu \mathrm{L}$ of culture medium. The medium in the wells was exchanged with fresh medium when the GCs reached $60 \%$ confluence and transfection was started subsequently. The plate was pre-incubated in an incubator $\left(37^{\circ} \mathrm{C}, 5 \% \mathrm{CO} 2\right)$ for $0,12,24,48$ and $72 \mathrm{~h}$. The CCK- 8 (TaKaRa, Dalian, China) reagents $(10 \mu \mathrm{L})$ was then added to each well. The plate was incubated for $30 \mathrm{~min}$. The optical density in each well was measured at $450 \mathrm{~nm}$ using a plate reader (Tecan Infinite ${ }^{\oplus} 200$, Tecan Life Sciences, Männedorf, Switzerland) calibrated to a blank well. For each group, the mean value of three wells was obtained to determine the proliferation curve.

\section{Bioinformatics analysis}

Three online software options were used to predict target miRNAs for the FSHR 3' UTR: TargetScan (http://www.targetscan. org/vert_71/), miRNAMAP (http://mirnamap.mbc.nctu.edu.tw/) and miRCode (http://www.mircode.org/). The mature sequences of miR-31 were obtained from miRBase (http://www.mirbase.org/).

Table 1. Primers in this study

\begin{tabular}{|l|l|c|}
\hline \multicolumn{1}{|c|}{ Name } & \multicolumn{1}{|c|}{ Primer sequences (5'-3') } & \multicolumn{1}{c|}{ Application } \\
\hline FSHR 3'UTR & $\begin{array}{l}\text { F:CGAGCTCATCTCCTTCTTTGCCATCTC } \\
\text { R:CCGCTCGAGGGATGTGCCAGGGAGAT }\end{array}$ & Vector construct \\
\hline FSHR CDS Region & $\begin{array}{l}\text { F:GGATCCTCAAGATAATATAAACATCCACACA } \\
\text { R:CTCGAGATGAAGTATGTGGAAGTGCTCTGTC }\end{array}$ & Vector construct \\
\hline FSHR 3'UTR MT & $\begin{array}{l}\text { F:GCTGACAGAGCACTTCCACATACTTCATCTAATTT } \\
\text { R:ATATTCTTCAAAGGCAATCATTATCTGTCATTCAA }\end{array}$ & Mutation Vector construct \\
\hline FSHR 3'UTR MT & $\begin{array}{l}\text { F:TGACAGAGCACTTCCACATACTTCATCTAATTTAA } \\
\text { R:GCATATTCTTCAAAGGACCGACTTATCTGTCATTC }\end{array}$ & qRT-PCR \\
\hline FSHR & $\begin{array}{l}\text { F:GCTTTTGCAGTTGCCCTCT } \\
\text { R:ATTGAGCACAAGGAGGGACAT }\end{array}$ & qRT-PCR \\
\hline PCNA & $\begin{array}{l}\text { F:TCCGAGGGCTTCGACAC } \\
\text { R:AGGCATCTTTACTACACAGCTATAC }\end{array}$ & qRT-PCR \\
\hline GAPDH & $\begin{array}{l}\text { F:CGGGCGCCTGGTCAC } \\
\text { R:ACTTGCCGTGGGTGGAATC }\end{array}$ \\
\hline
\end{tabular}

Digestion sites are underlined 
Table 2. Small fragments of RNA synthesized in the present study

\begin{tabular}{|l|l|}
\hline \multicolumn{1}{|c|}{ Name } & Sequences (5'-3') \\
\hline miR-31 mimics & AGGCAAGAUGCUGGCAUAGCU \\
\hline miR-23a mimics & GGGGUUCCUGGGGAUGGGAUUU \\
\hline miR-23b mimics & UGGGUUCCUGGCAUGCUGAUUU \\
\hline miR-144 mimics & GGAUAUCAUCAUAUACUGUAAG \\
\hline miR-203 mimics & AGUGGUUCUUGACAGUUCAACA \\
\hline miR-33a mimics & GUGCAUUGUAGUUGCAUUGCA \\
\hline miR-33b mimics & GUGCAUUGCUGUUGCAUUGC \\
\hline miR-512-5p mimics & CACUCAGCCUUGAGGGCACUUUC \\
\hline miR-92a mimics & UAUUGCACUUGUCCCGGCCUGU \\
\hline miR-363 mimics & AUUGCACGGUAUCCAUCUGCG \\
\hline mimics NC & UUGUUCAAACAAUAAGUAGUC \\
\hline mimics inhibitor & CUGCCUUGACGAUUUCGUUUU \\
\hline Inhibitor NC & CUTACCUTGACGGCAUTGGCAT \\
\hline siRNA-1 & CCUAUACUCGAUAAAGUUUTT \\
\hline siRNA-2 & AAGCUUUGUGGUGUAUAGGTT \\
\hline siRNA Control & GGCCAUUUGGAUAUAAUUTT \\
\hline
\end{tabular}

\section{RNA isolation and RT-PCR}

Total RNA was extracted from GCs and HEK 293T cells using the TRIzol Reagent (Invitrogen) and reverse transcribed using the PrimeScriptTM RT Master Mix (TaKaRa). Quantitative real-time PCR (RT-PCR) was performed using the SYBR Green Master Mix (Vazyme Biotech, Nanjing, China) and the mRNA relative expression levels were calculated using the $2^{-\Delta \Delta} \mathrm{Ct}$ method. Primer sequences are listed in Table 1.

\section{Radioimmunoassay}

The GCs medium was changed to serum-free medium when measuring progesterone levels. After transfection for $48 \mathrm{~h}$, the cell culture medium was collected and the concentration of P4 was analyzed using the commercially available Iodine[125I] Progesterone Radioimmunoassay Kit (Xinfan Biological Company, Shanghai, China), according to the manufacturer's instructions. Samples are expressed as $\mathrm{ng} / \mathrm{mL}$. Each group has three replicas.

\section{BMP15 treatment}

Yak GCs were seeded in six-well plates at a density of $1 \times 10^{6}$ cells per well and cultured in medium containing 15\% FBS for $24 \mathrm{~h}$. The cell culture medium was then replaced with fresh medium that contain different concentrations of BMP15. After 0-72 h, cells and the cell culture medium were collected, and the secretion of P4, GCs proliferation and FSHR expression were examined.

\section{Statistical analysis}

Statistical analysis was conducted by SPSS V20.0 (SPSS, Chicago, IL, USA). All data are presented as the mean \pm SEM (standard error of the mean). SEM standard deviation of sample mean was a scale describing the dispersion of mean sampling distribution and measuring the magnitude of mean sampling error, reflecting the variation between sample mean. The range of mean \pm SEM as an indicator symbol to mark which ones have been removed. Each experiment consists of three biological repetitions and repeates three times. The control group and treatment group always have nine data respectively, the range of mean \pm SEM was used to discard data, making the data approximate normal distribution. Generally speaking, if there are big difference between a set of data, it was necessary to do the experiment again so as to ensure the accuracy of data. Then the unpaired two-tailed Student's t-test and ANOVA were used to evaluate the statistical significance of the differences and $\mathrm{P}<$ 0.05 was considered statistically significant.

\section{Results}

BMP15 regulates GC proliferation and secretion of P4 and expression of FSHR in yak ovarian

BMP15 regulates the development of animal follicles, including the promotion of GC proliferation, diffusion of the cumulus and regulation of the production of steroid hormones such as progesterone in GCs [26-29]. Yak GCs were treated with different concentrations of BMP15 to investigate how BMP15 regulates GC proliferation and hormone secretion in yak ovaries. The proliferation of yak GCs was detected using the CCK- 8 kit. The results showed that the proliferation of yak GCs increased significantly (5.3 times) at $100 \mathrm{ng} / \mathrm{mL}$ (Figure 1A). The results are similar to previous studies $[30,31]$. We also explored the effect of BMP15 on proliferation of yak GCs at different time points. The results showed that proliferation of yak GCs increased from 0-72 h after treatment with BMP15, and the difference in proliferation to that of the control sample increased as the incubation time increased (Figure 1B). We treated GCs with different concentrations of BMP15 and $1 \mu \mathrm{mol} / \mathrm{mL}$ BMP15 inhibitor-K02288 (ALK6; $1 \mu \mathrm{mol} / \mathrm{mL}$, which is the most suitable concentration of K02288) for $48 \mathrm{~h}$ to further explore the regulatory effect of BMP15 on the secretion of P4 in yak GCs. There was no significant difference between the control group and the BMP15 treated sample from 0 to $50 \mathrm{ng} / \mathrm{mL}$ (Figure 1C). P4 synthesis was inhibited 1.5-fold when the concentration of BMP15 was $100 \mathrm{ng} / \mathrm{mL}$. Treatment at $100 \mathrm{ng} / \mathrm{mL}$ caused significant (i.e., 5-fold) inhibition of P4 synthesis in GCs. The inhibitory effect of BMP15 on the secretion 
of P4 by yak GCs was removed following treatment of the yak GCs with the BMP15 signaling pathway inhibitor K02288 (Figure 1C). Moreover, BMP15 was found to have the same inhibitory effect on FSHR (Figure 1D). These observations suggest that BMP15 may regulate the proliferation of GCs, and P4 synthesis secretion through FSHR.

\section{Knockdown of FSHR promotes GC proliferation in yak ovarian}

Previous studies have shown that the mRNA levels of FSHR are dependent on the size of ovarian GCs [32]. Thus, we hypothesized that yak ovarian GC proliferation is due to differences in the expression level of FSHR because of BMP15 regulation. Two FSHR-specific small interfering RNAs (siRNAs) were designed (named F-siRNA-1 and F-siRNA-2) and transfected into yak GCs to test the role of FSHR in yak GC proliferation. We found that F-siRNA-1 dramatically decreased the expression of FSHR at both the mRNA and protein levels (Figures 2A and 2B). Additionally, yak GC proliferation and PCNA mRNA expression were induced significantly following FSHR knockdown (Figures 2C and 2D).
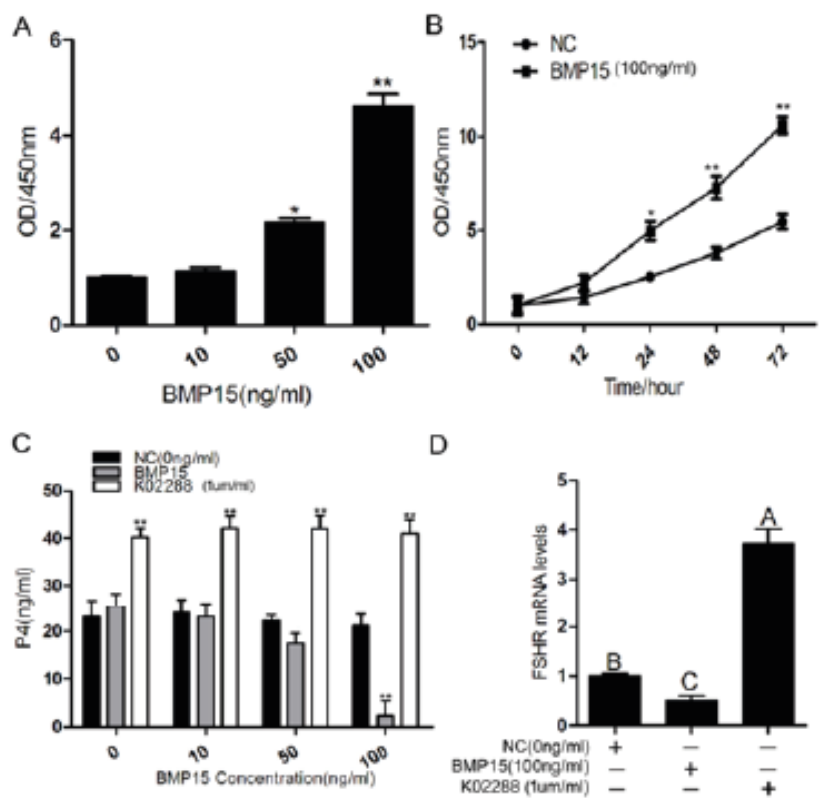

Figure 1. BMP15 regulates GC proliferation, secretion of $P 4$ and expression of FSHR in yak ovaries. (A) Yak GC proliferation levels transfected with different concentrations of BMP15. (B) The proliferation curve of yak GCs. (C) Effect of different concentrations of BMP15 and $1 \mu M$ KO2288 on the secretion of P4. (D) Effect of BMP15 $(100 \mathrm{ng} / \mathrm{mL})$ and KO2288 (1 $\mu \mathrm{M})$ on the expression of FSHR. All experiments were performed in triplicate and the data presented represent the mean $\pm S E M$. $* P<0.05 ; * * P<0.01$.

Overexpression of FSHR inhibits proliferation of GCs in yak ovarian

pcDNA3.1-FSHR was transfected into yak GCs to further investigate the role of FSHR in yak GC proliferation. We found that pcDNA3.1-FSHR dramatically increased the expression of FSHR at both the mRNA and protein levels (Figures 3A and 3B). Moreover, using the CCK- 8 kit revealed that overexpression of FSHR reduced the proliferation rate of yak GCs (Figure 3C), and the expression of PCNA mRNA was dramatically reduced (Figure 3D). These aforementioned observations demonstrate that FSHR regulates the proliferation of yak GCs, which is regulated by BMP15.
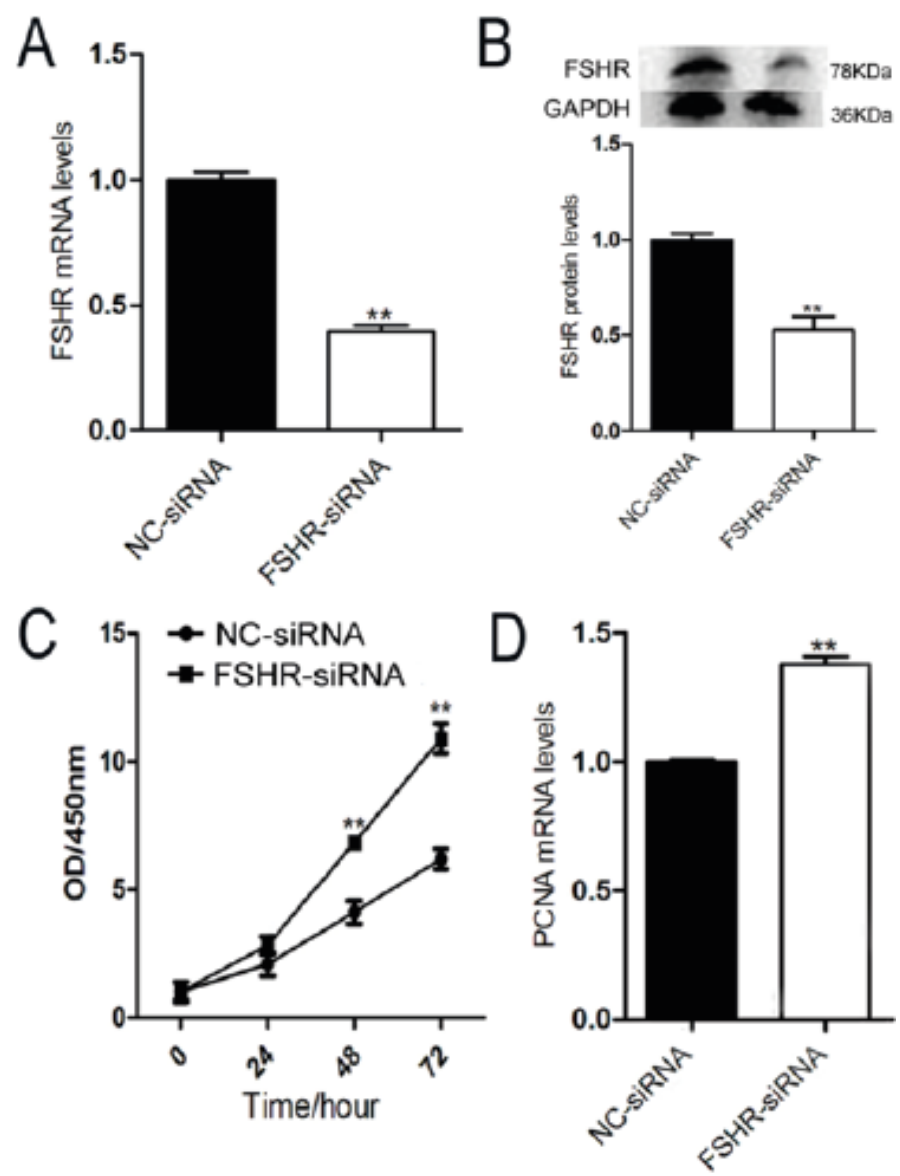

Figure 2. Knockdown of FSHR promotes proliferation of yak GCs. Knockdown of FSHR decreases its $m R N A$ (A) and protein (B) levels. Knockdown of FSHR promotes proliferation of yak GCs (C) and PCNA mRNA (D) expression. All experiments were performed in triplicate and the data presented represent the mean $\pm S E M$. ${ }^{*} P<0.01$.

\section{FSHR is a direct target of miR-31}

Recent studies have shown that miRNAs, as an epigenetic regulator, have a regulatory effect on ovarian development, follicle growth and ovarian GC development. We postulated that BMP15 may affect FSHR by regulating the expression of miRNAs, which would, in turn, regulate the proliferation of GCs and secretion of P4 by yak GCs. To explore this hypothesis, we identified miRNAs that target FSHR and are regulated by BMP15 in yak GCs (Figure 4A). A total of 10 candidate miRNAs were predicted to target FSHR (Figure 4B). After treatment of GCs with $100 \mathrm{ng} / \mathrm{mL} \mathrm{BMP15,} \mathrm{the}$ expression level of the 10 miRNAs were detected, and miR-31 was significantly upregulated (Figure 4C). Therefore, we focused on miR31 in our subsequent analyses. Sequence alignment revealed that vertebrate miR-31 genes share a common seed sequence (Figure 4D). This seed sequence is complementary to sequences present in the $3^{\prime}$ UTRs of FSHR in bovine, human, pig and chimpanzees (Figure 4E). We constructed luciferase reporters of the yak FSHR 3' UTR that contained the putative miR-31 target sites and mutant forms of the target sites to determine whether the FSHR is a true target of miR-31 (Figure 4F). The results of the luciferase assays showed that the wildtype vector was reduced markedly upon co-transfection with miR-31 mimic, but no change was observed for the mutant reporter (Figure $4 \mathrm{G})$. These results demonstrate that FSHR is a direct target of miR-31.

\section{miR-31 regulates the expression of FSHR}

The expression level of FSHR was detected by $\mathrm{qPCR}$ and western blotting analysis after transforming cells with the miR-31 mimic and miR-31 inhibitor to further determine whether miR-31 

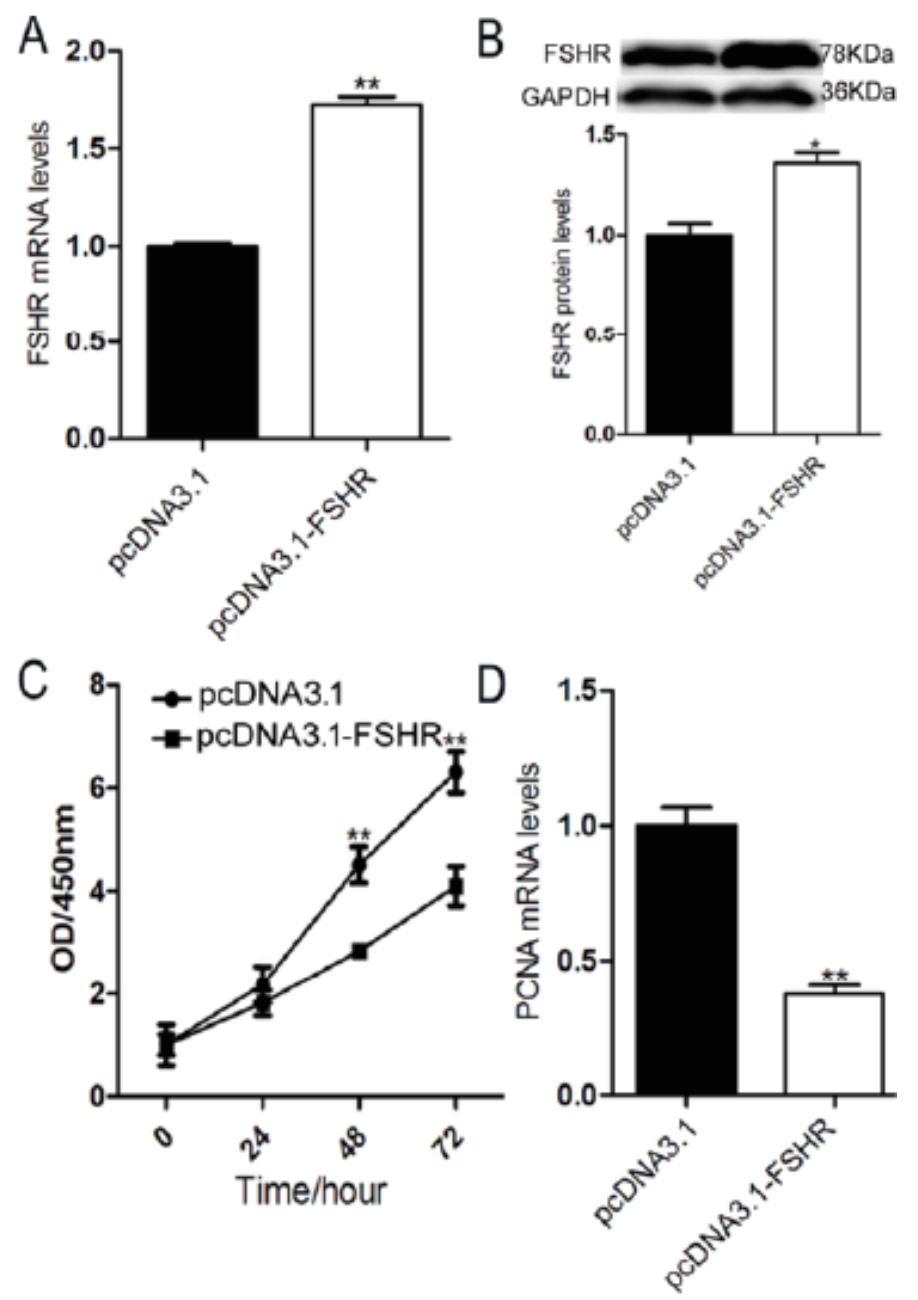

Figure 3. Overexpression FSHR inhibits proliferation of yak GCs. Overexpression of FSHR increases its $m R N A(A)$ and protein $(B)$ levels. Overexpression of FSHR inhibits proliferation of yak GCs (C) and PCNA mRNA (D) expression. All experiments were performed in triplicate and the data presented represent the mean $\pm S E M$. $* P<$ $0.05 ; * * P<0.01$.

regulates endogenous FSHR expression. The results showed that the FSHR mRNA and protein levels decreased significantly in yak GCs after transfection with the miR-31 mimic (Figures 5A and 5B) and increased significantly in yak GCs after transfection with the miR31 inhibitor (Figures 5C and 5D), revealing that miR-31 can directly target FSHR and regulate FSHR expression in yak GCs.

miR-31 induces cell proliferation in yak GCs by targeting FSHR

We transfected miR-31 mimic into the yak GCs to explore how miR-31 regulates proliferation of yak GCs. The results from a CCK- 8 experiment showed that overexpression of miR-31 in yak GCs induce cell proliferation (Figure 6A). qR

T-PCR showed that PCNA mRNA levels were remarkably increased (Figure 6B). These data suggest that miR-31 is a regulator of GC proliferation. We then co-transfected miR-31 mimic and pcDNA3.1-FSHR into yak GCs to further determine whether miR-31 regulates GC proliferation by targeting FSHR. The results showed that overexpression of FSHR rescues miR-31-induced GC proliferation (Figure 6C), and PCNA mRNA levels also decreased (Figure 6D). These results demonstrate that miR-31 regulates yak GC proliferation by targeting FSHR.
A
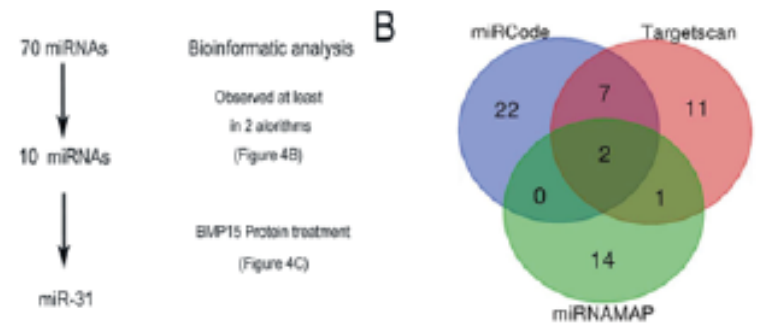

C

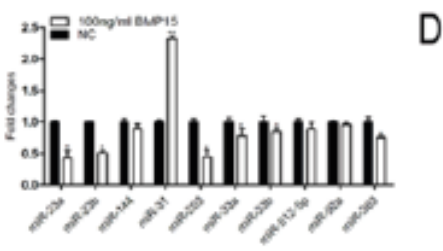

$\mathrm{E}$ $\operatorname{miR}-31$

Seed reounces

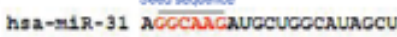
MnU-M1R-31 ACCCAMCAUGCUCCCAUACCU gga-M1R-31 ACCCANCAUGUUCOCAUACCU men1-M1R-31 ACCCANCAUGCUCCCAUAGCU

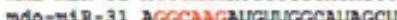
beno-

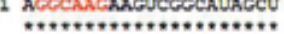

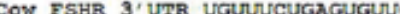

GACAGAUNA-

GaAUGAUAAUUAARGUCUUGGCCIUUGA HUman FSHR 3' UTR UGUAUCUGAGUAUUGANUGNUNAUUCA-GUCUUGGCCYYUGA Chimp ISHR 3'UTR UGUAUCUGAGUGUUGARUGAUAAUUCA-AUCUUGCCUUUGA $* * * * * * * * * * * * * * * * * * * * * * * * * * * * * * *$

$\mathrm{F}$
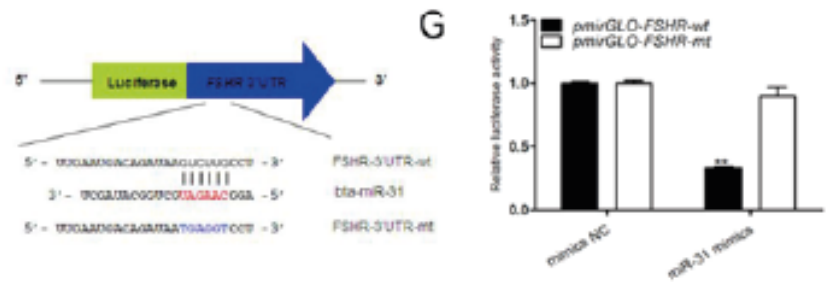

Figure 4. FSHR is a target of miR-31, which is regulated by BMP15. (A) A schematic diagram of the strategy used to identify miRNAs targeting FSHR and regulated by BMP15. (B) miRNAs targeting FSHR were predicted by three different algorithms. (C) BMP15 promotes miR-31 expression in yak GCs. miR-31 levels were examined by $q R T$ $P C R$ and U6 was used as the control. (D) Alignment of sequences of mature miR-31 from six vertebrates. Letters in red indicate the seed sequence. (E) Comparison of miR-31 seed regions and the FSHR 3' UTRs from bovine, pig, human and chimpanzee. Asterisks indicate complementarity. (F) miR-31 binding sites in wild-type and mutant constructs. A putative miR-31 binding site pair with the miR-31 seed region (red letters); mutated binding sites are shown in blue. (G) Luciferase activity assays. pmirGLO-FSHR-3'-UTR wild-type and pmirGLO-FSHR-3'UTR mutant were co-transfected into $293 T$ cells along with miR-31 and the NC mimic. All data are presented as the mean $\pm S E M,(n=3)$. $* P$ $<0.05 ; * * P<0.01$.

miR-31 regulates GCs proliferation and P4 secretion by targeting FSHR, which is regulated by BMP15

The mechanism of BMP15 regulation of yak GC proliferation and P4 inhibition was examined. We first treated yak GCs with $100 \mathrm{ng} / \mathrm{mL}$ BMP15. The secretion of P4 in the culture medium of yak GCs was reduced significantly when compared with that of the control group (Figure 7A), and the mRNA (Figure 7B) and protein (Figure 7C) expression levels of FSHR were significantly inhibited. In addition, co-overexpression of miR-31 and BMP15 in yak GCs caused a further two-fold reduction in secretion of P4 and FSHR expression (Figure 7). Moreover, treatment with the BMP15 inhibitor K02288 alleviated the inhibition of GC proliferation and $\mathrm{P} 4$ secretion. This observation suggests that BMP15 may directly affect FSHR and inhibit its expression. Our results herein have shown that miR-31 targets the FSHR. Thus, we used BMP15 and the miR-31 inhibitor to treat yak GCs simultaneously. The results showed that the inhibitory effect of BMP15 and miR-31 inhibitor on the secretion of P4 and the expression of FSHR were reduced significantly (Fig. 7) when 

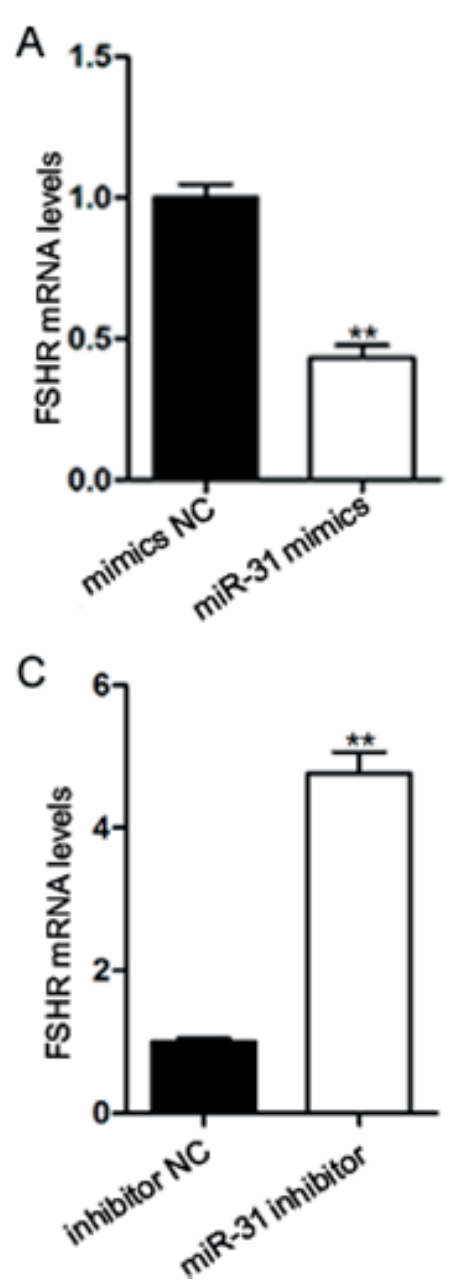
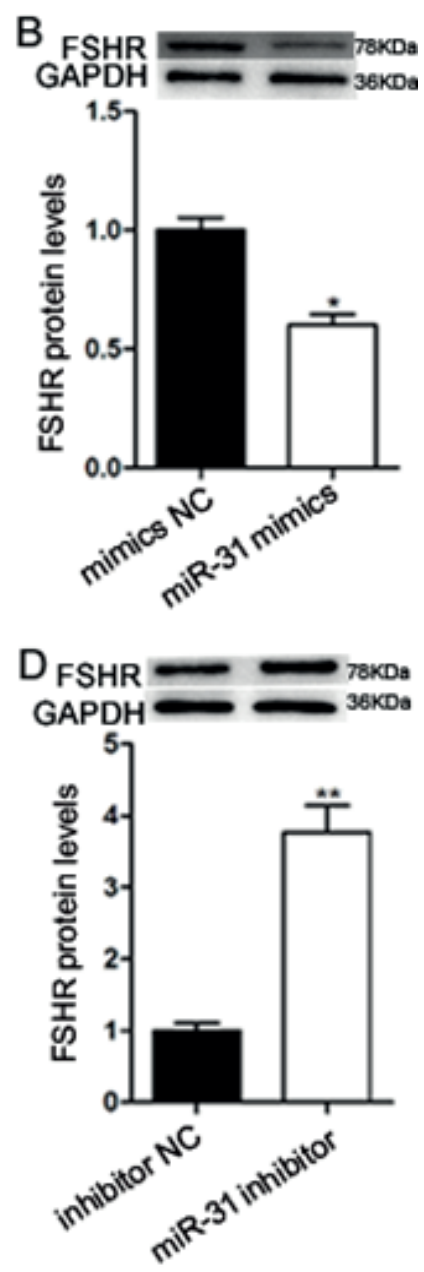

Figure 5. miR-31 repression of FSHR in yak GCs. Overexpression of miR-31 decreases FSHR mRNA (A) and protein (B) levels. miR-31 or $N C$ mimic were transfected into cultured yak GCs. FSHR $m R N A$ and protein levels were detected and normalized to GAPDH. Inhibition of miR-31 increases FSHR mRNA (C) and protein (D) levels. All data presented represent the mean $\pm S E M,(n=3)$. $* P<0.05$; $* * P<0.01$.

compared with the results of BMP15 treatment only, revealing that miR-31 regulates GC proliferation and secretion of $\mathrm{P} 4$ via reducing FSHR expression, which is regulated by BMP15.

\section{Discussion}

Follicle stimulating hormone is essential for normal follicle formation and female reproduction [33]. However, FSH is a biological macromolecule and cannot readily cross the cell membrane [34]. Thus, entry into a cell must be mediated by FSHR, which is specifically expressed on the membrane of GCs found in the ovary [34]. The level of FSH in cells is also regulated by FSHR expression and activity levels [35]. FSHR is a member of the G protein coupled receptor family and mediates FSH signal transduction through the cAMP pathway [36]. Studies have shown that FSHR mRNA is expressed in GCs and oocytes from ovaries [37]. Moreover, the expression of FSHR mRNA in the different follicle and GC development stages varies significantly because of the different degrees of dependence on FSH [32,38]. Thus, in the process of mammalian ovarian follicle development the stimulation of FSH toward GCs needs to be combined with FSHR, and the amount of FSHR expressed on the surface of cells regulates the response of cells toward FSH. In addition, there is strong evidence that regulatory factors secreted by oocytes can affect the activity of FSH and FSHR $[13,39]$. In recent years, an oocyte growth factor BMP15, which is involved in the regulation of FSH, has attracted significant
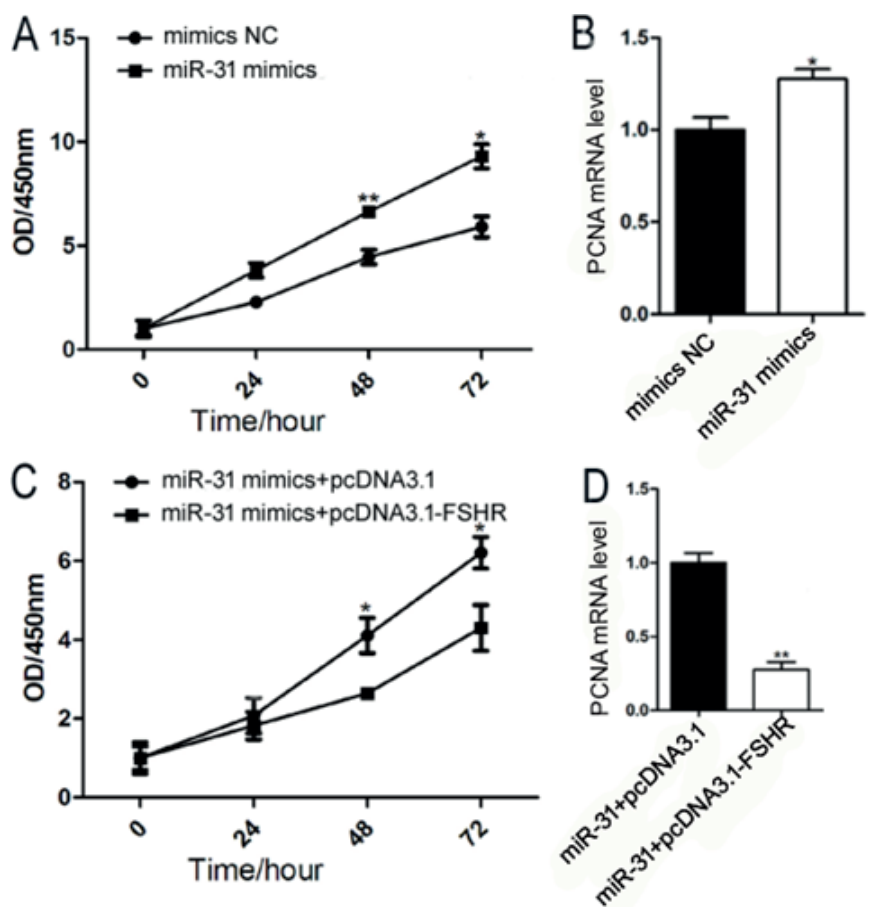

Figure 6. miR-31 promotes the proliferation of yak GCs by targeting FSHR. Overexpression of miR-31 affected the proliferation of yak GCs (A) and PCNA mRNA (B) expression. Co-overexpression of miR31 and FSHR affected the proliferation of yak GCs (C) and PCNA $m R N A(D)$ expression. All data presented represent the mean $\pm S E M$, $(n=3) . * P<0.05 ; * * P<0.01$.
A

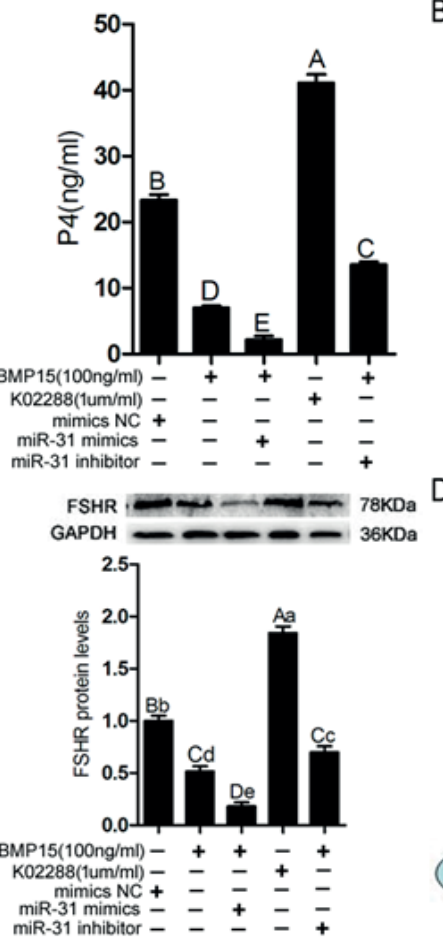

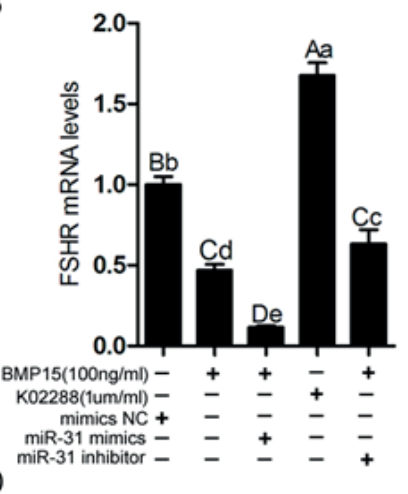

Figure 7. The effect of BMP15 and miR-31 on FSHR and secretion of $P 4$ in yak GCs. (A) Effect of BMP15 and miR-31 on the secretion of P4 in yak GCs. (B) Effect of BMP15 and miR-31 on FSHR mRNA (B) and protein $(C)$ levels in yak GCs. (D) Signal path diagram. All data presented represent the mean $\pm S E M,(n=3)$. $* P<0.05 ; * * P<0.01$. 
attention. In a study examining the ovulation rate of sheep, BMP15 was identified to play a role in promoting cell proliferation and differentiation of follicle cells [40]. BMP15 was identified as a negative regulator of rat ovary FSH. Otsuka and colleagues demonstrated that BMP15 downregulates FSH by inhibiting the expression of FSHR, promotes the proliferation of GC in rats and downregulates FSHinduced secretion of P4 [27,41]. Similarly, our results clearly indicated that BMP15 completely inhibited the activities of FSHR in yak GCs. The expression of FSHR mRNA was inhibited significantly when yak GCs were treated with BMP15. In addition, when the BMP15 signal pathway was suppressed by treatment with ALK6, the expression of FSHR mRNA was promoted significantly. Moreover, after knockingdown FSHR, the proliferation of yak GCs and PCNA mRNA expression were promoted significantly, and overexpression of FSHR resulted in the opposite effects. We hypothesize that BMP15 promotes the proliferation of yak GCs by inhibiting the expression of FSHR. Moreover, we measured the secretion of P4 by yak GCs and observed that a $100 \mathrm{ng} / \mathrm{mL}$ treatment with BMP15 caused significant reduction of P4 secretion by this cell type. There is also substantial evidence showing that many regulatory factors inhibit GC luteinization during oocyte development [42]. This indicates that BMP15 determines the effect of FSH in GCs by inhibiting FSHR expression.

The development of GCs and secretion of hormones are regulated by many factors. In recent years, it was reported that miRNA may play an important role in regulating GC proliferation, apoptosis and the secretion of gonadal hormones $[22,43,44]$. Yao et al. treated GCs for $12 \mathrm{~h}$ with FSH, and showed the upregulation of 17 miRNAs and the downregulation of 14 miRNAs, which led to an increase in progesterone secretion [45]. Sirotkin et al. used gene chip technology (genome-scale) to identify 51 miRNAs, including miR-31, that inhibit the secretion of progesterone, androgen and estrogen [46]. In this study, we found that miR-31 reduced FSHR expression and promoted the proliferation of yak GCs by directly combining with the 3' UTR of FSHR. We evaluated the function of the BMP15/miR31 axis and FSHR in regulating GC proliferation and secretion of P4. We first treated yak GCs with $100 \mathrm{ng} / \mathrm{mL}$ BMP15. Compared with the transfected control cells, the level of $\mathrm{P} 4$ in the culture medium of yak GCs treated with BMP15 was clearly reduced, and the mRNA and protein expression levels of FSHR were reduced significantly. In addition, simultaneous treatment of yak GCs with miR-31 and 100 $\mathrm{ng} / \mathrm{mL}$ BMP15 caused a further reduction of P4 secretion and FSHR inhibition was enhanced by two-fold. Transfection with the BMP15 inhibitor K02288 removed the inhibitory effect of BMP15 and FSHR activity was promoted. We also observed that the inhibitory effects on the secretion of P4 and the expression of FSHR by BMP15 and the miR-31 inhibitor were significantly reduced when compared with that of the BMP15-treated GCs.

\section{Conclusion}

This study found that BMP15 could mediate FSHR regulated by miR-31 to regulate the proliferation of yak GCs and P4 secretion.

\section{Acknowledgements}

The publication charge was supported by the National Beef Cattle and Yak Industry System, Ministry of Agriculture of the Republic of China [grant number CARS-37], and other works was supported by the National Natural Science Foundation of China [grant number 31460604;31960661], Key Projects of the Science and Technology Department of Tibet Autonomous Region [grant number: 2019].

\section{Competing interests}

The authors declare that there is no conflict of interests regarding the publication of this article.

\section{Author contribution}

Yilong Yao, Yuheng Wang and Yun Chen were mainly contributed to do experiments; Qifa Li and Linghua Cheng were contributed to the conception of the study; Jiaqiang Niu, Honghui Wang and Suolang Sizhu were contributed significantly to analysis and manuscript preparation; Yefen Xu was performed the data analyses and wrote the manuscript; Qiangba Yangzong and Min Guo were helped perform the analysis with constructive discussions.

\section{References}

1. Pernasetti F, Spady TJ, Hall SB, et al. Pituitary tumorigenesis targeted by the ovine follicle-stimulating hormone $\beta$-subunit gene regulatory region in transgen ic mice. Mol Cell Endocrinol. 2003;203:169-183.

2. Costa SLD, Costa EPD, Pereira ECM, et al. Structural characterization of the follicle stimulating hormone and its role in the physiology of ovarian cells. Ci Anim. 2014; 24(1):11-23.

3. Valenti D, Vignera SL, Condorelli RA, et al. Follicle-stimulating hormone treatment in normogonadotropic infertile men. Nat Rev Urol. 2013;10:55-62.

4. Miras AD, Mogford JT, Wright J, et al. Ovarian hyperstimulation from ectopic hypersecretion of follicle stimulating hormone. Lancet. 2015;385:392-392.

5. Rybska M, Knap S, Jankowski M, et al. Characteristic of factors influencing the proper course of folliculogenesis in mammals. Med J Cell Biol. 2018;6:33-38.

6. Nielsen ME, Rasmussen IA, Kristensen SG, et al. In human granulosa cells from small antral follicles, androgen receptor mrna and androgen levels in follicular fluid correlate with fsh receptor mrna. Mol Hum Reprod. 2011;17:63-70.

7. Zhang S, Li W, Zhu C, et al. Sertoli cell-specific expression of metastasis-associated protein $2(\mathrm{mta} 2)$ is required for transcriptional regulation of the follicle-stimulating hormone receptor (fshr) gene during spermatogenesis. J Biol Chem. 2012;287:40471-40483.

8. $\mathrm{Wu} \mathrm{W}, \mathrm{Han} J$, Cao R, et al. Sequence and regulation of the porcine fshr gene promoter. Anim Reprod Sci. 2015;154:95-104.

9. Mazurkiewicz JE, Herrickdavis K, Barroso M, et al. Singlemolecule analyses of fully functional fluorescent protein-tagged follitropin receptor reveal homodimerization and specific heterodimerization with lutropin receptorl. Biol Reprod. 2015;92:100.

10. Wei S, Chen S, Gong Z, et al. Alarelin active immunization influences expression levels of gnrhr, fshr and lhr proteins in the ovary and enhances follicular development in ewes. Anim Sci J. 2013;84:466-475.

11. Sriraman V, Denis D, de Matos D, Yu H, Palmer S, Nataraja $\mathrm{S}$. Investigation of a thiazolidinone derivative as an allosteric modulator of follicle stimulating hormone receptor: Evidence for its ability to support follicular development and ovulation. Biochem Pharmacol. 2014;89:266-275.

12. Sen A, Hammes SR. Granulosa cell-specific androgen receptors are critical regulators of ovarian development and function. Mol Endocrinol. 2010;24:1393-1403.

13. Matzuk MM. Revelations of ovarian follicle biology from gene knockout mice. Mol Cell Endocrinol. 2000;163:61-66.

14. Eppig JJ, Chesnel F, Hirao Y, et al. Oocyte control of granulosa cell development: How and why. Hum Reprod. 1997;12:127-132.

15. Goldschmit D, Kraicer P, Orly J. Periovulatory expression of cholesterol side-chain cleavage cytochrome $\mathrm{p}-450$ in cumulus cells. Endocrinology. 1989;124:369.

16. Vanderhyden BC, Tonary AM. Differential regulation of progesterone and estradiol production by mouse cumulus and mural granulosa cells by a factor(s) secreted by the oocyte. Biol Reprod. 1995;53:1243-1250.

17. Galloway SM, McNatty KP, Cambridge LM, et al. Mutations in an oocyte-derived growth factor gene (bmp15) cause increased ovulation rate and infertility in a dosage-sensitive manner. Nat Genet. 2000;25:279-283.

18. Dube JL, Wang P, Elvin J, Lyons KM, Celeste AJ, Matzuk MM. The bone morphogenetic protein 15 gene is $x$-linked and expressed in oocytes. Mol Endocrinol. 1998;12:1809-1817.

19. Otsuka F, Yao Z, Lee T, Yamamoto S, Erickson GF, Shimasaki $S$. Bone morphogenetic protein-15. Identification of target cells and biological functions. J Biol Chem. 2000;275:39523-39528. 
20. Otsuka F, Yamamoto S, Erickson GF, Shimasaki S. Bone morphogenetic protein-15 inhibits follicle-stimulating hormone (fsh) action by suppressing fsh receptor expression. J Biol Chem. 2001;276:11387-11392.

21. Imbar T, Eisenberg I. Regulatory role of micrornas in ovarian function. Fertil Steril. 2014;101:1524-1530.

22. Liu J, Tu F, Yao W, et al. Conserved mir-26b enhances ovarian granulosa cell apoptosis through has2-ha-cd44-caspase-3 pathway by targeting has2. Sci Rep. 2016;6:21197.

23. Du X, Li Q, Pan Z, Li Q. Androgen receptor and mirna-126* axis controls follicle-stimulating hormone receptor expression in porcine ovarian granulosa cells. Reproduction. 2016;152:161.

24. Cui X, Jing X, Wu X, et al. Abnormal expression levels of bmp15/ smad1 are associated with granulosa cell apoptosis in patients with polycystic ovary syndrome. Mol Med Rep 2017; 16(6):82318236.

25. Du X, Zhang L, Li X, Pan Z, Liu H, Li Q. Tgf-beta signaling controls fshr signaling-reduced ovarian granulosa cell apoptosis through the smad4/mir-143 axis. Cell Death Dis. 2016;7:e2476.

26. Montgomery GW, Galloway SM, Davis GH, Mcnatty KP. Genes controlling ovulation rate in sheep. Reproduction. 2001;121:843852.

27. Otsuka F, Yao Z, Lee T, Yamamoto S, Erickson GF, Shimasaki $\mathrm{S}$. Bone morphogenetic protein-15. Identification of target cells and biological functions. J Biol Chem. 2000;275:39523-39528.

28. Epifano O, Dean J. Genetic control of early folliculogenesis in mice. Trends Endocrinol Metab. 2002;13(4):169-173. Paulini F, Melo EO. The role of oocyte-secreted factors gdf9 and bmp15 in follicular development and oogenesis. Reprod Domest Anim. 2011;46:354-361.

29. Sudiman J, Sutton-Mcdowall ML, Ritter LJ, et al. Bone morphogenetic protein 15 in the pro-mature complex form enhances bovine oocyte developmental competence. Plos One. 2014;9:e103563.

30. Hosoe M, Kaneyama K, Ushizawa K, Hayashi K, Takahashi T. Quantitative analysis of bone morphogenetic protein 15 (bmp15) and growth differentiation factor 9 (gdf9) gene expression in calf and adult bovine ovaries. Reprod Biol Endocrin. 2011;9:33-33.

31. Liu J, Aronow BJ, Witte DP, Pope WF, La Barbera AR. Cyclic and maturation-dependent regulation of follicle-stimulating hormone receptor and luteinizing hormone receptor messenger ribonucleic acid expression in the porcine ovary. Biol Reprod. 1998;58:648-658.

32. Grazul-Bilska AT, Reyaz A, Valkov V, Dorsam ST, Redmer DA. Follicle stimulating hormone receptor protein is expressed in ovine uterus during the estrous cycle and utero-placenta during early pregnancy: An immunohistochemical study. Acta
Histochemica 2018;120(5): 420-428

33. Huhtaniemi IT. Ontogeny of follicle-stimulating hormone receptor gene expression in the rat testis and ovary. Mol Cell Endocrinol. 1995;107:199-208.

34. Tilly JL, LaPolt PS, Hsueh AJ. Hormonal regulation of folliclestimulating hormone receptor messenger ribonucleic acid levels in cultured rat granulosa cells. Endocrinology. 1992;130:12961302.

35. Braun T, Schofield PR, Sprengel R. Amino-terminal leucinerich repeats in gonadotropin receptors determine hormone specificity. Embo j 10: 1885-1890. Embo Journal. 1991;10:18851890.

36. Zachos NC, Billiar RB, Albrecht ED, Pepe GJ. Developmental regulation of follicle-stimulating hormone receptor messenger rna expression in the baboon fetal ovary. Biol Reprod. 2003;68:1911.

37. Drion PV, Beckers JF, Derkenne F, Hanzen C. Follicular development in the cow: Hormonal regulations involved in the control of estrous cycle and post-partum period. Annales De Médecine Vétérinaire 2000;144:385-402.

38. Eppig, JJ, Chesnel, Hirao, O'Brien, MJ, Pendola, FL. Oocyte control of granulosa cell development: How and why. Hum Reprod. 1997;12:127-132.

39. Zamani P, Nadri S, Saffaripour R, Ahmadi A, Dashti F, Abdoli R. A new mutation in exon 2 of the bone morphogenetic protein 15 gene is associated with increase in prolificacy of mehraban and lori sheep. Trop Anim Health Prod. 2015;47:855-860.

40. Otsuka F, Yamamoto S, Erickson GF, Shimasaki S. Bone morphogenetic protein-15 inhibits follicle-stimulating hormone (fsh) action by suppressing fsh receptor expression. J Biol Chem. 2001;276:11387-11392.

41. Pescador N, Stocco DM, Murphy BD. Growth factor modulation of steroidogenic acute regulatory protein and luteinization in the pig ovary. Biol Reprod. 1999;60:1453.

42. Lei L, Jin S, Gonzalez G, Behringer RR, Woodruff TK. The regulatory role of dicer in folliculogenesis in mice. Mol Cell Endocrinol. 2010;315:63-73.

43. Torley KJ, da Silveira JC, Smith P, et al. Expression of mirnas in ovine fetal gonads: Potential role in gonadal differentiation. Reprod Biol Endocrinol. 2011;9:2.

44. Yao N, Yang BQ, Liu Y, et al. Follicle-stimulating hormone regulation of microrna expression on progesterone production in cultured rat granulosa cells. Endocrine. 2010;38:158-166.

45. Sirotkin AV, Ovcharenko D, Grossmann R, Laukova M, Mlyncek M. Identification of micrornas controlling human ovarian cell steroidogenesis via a genome-scale screen. J Cell Physiol. 2009;219:415-420. 